\title{
Relaxation Effect of a 2-Hour Walk in Kumano-Kodo Forest
}

\section{Teruhisa Komori ${ }^{1}$, Masayuki Mitsui ${ }^{2}$, Kenji Togashi ${ }^{3}$, Jun Matsui ${ }^{4}$, Takaya Kato ${ }^{5}$, Daisuke Uei ${ }^{5}$, Aritomo Shibayama ${ }^{6}$, Katsuhiro Yamato ${ }^{6}$ Hidehito Okumura ${ }^{6}$ and Fujihisa Kinoshita7}

\author{
${ }^{1}$ Department of Stress and Health Science, Mie Graduate School of Medicine, Mie, Japan \\ ${ }^{2}$ Mitsui Consulting, Osaka, Japan \\ ${ }^{3}$ Course of Health and Physical Education, Faculty of Education, Mie University, Mie, Japan, \\ ${ }^{4}$ Mie Technology Licensing Organization, Mie, Japan \\ ${ }^{5}$ Community-University Research Cooperation Center, Mie University, Mie, Japan \\ ${ }^{6}$ Owase City Hall, Owase, Japan \\ ${ }^{7}$ Laboratory of Kumano, Kumano-Kenko-Mura, Kumano, Japan
}

Corresponding author: Teruhisa Komori, Department of Stress and Health Science, Mie Graduate School of Medicine, Mie, Japan, Tel: +81 592315095, E-mail: komori@nurse.medic.mie-u.ac.jp

Received: Jan 25, 2017; Accepted: Feb 21, 2017; Published: Feb 23, 2017

Citation: Komori T, Mitsui M, Togashi K, et al. Relaxation Effect of a 2-Hour Walk in Kumano-Kodo Forest. J Neurol Neurosci. $2017,8: 1$.

\section{Abstract}

Background: Studies have shown that walking in forests can have a relaxation effect, but the participants only walked for about 15 minutes.

Methods and findings: The study adopted an open crossover design, in which 22 healthy male volunteers were randomly assigned to two groups. The first group walked the Kodo forest course first and the urban course second, while the second group completed the walks in the reverse order. The Kodo course was $4 \mathrm{~km}$ from the foot to the pass and back, and took around 2 hours. The participants walked the urban course at an exercise intensity equal to that of the Kodo course. We used both physiological and psychological measures to examine the relaxation effect of walking. Salivary amylase concentrations significantly increased from the start to the end of the urban course, and were significantly higher at the end of the urban course than at the end of the Kodo course. Saliva cortisol concentrations decreased from the start to the end of both courses, and were significantly lower at the end of the Kodo course than the urban course. Analysis of the Profile of Mood State data showed significant decreases in "Tension-Anxiety", "Depression-Dejection depression", "Anger-Hostility" and "Confusion" after walking both courses, and a significant decrease in "Vigor" after the urban course. Visual analog scores were significantly higher on the Kodo course, but decreased significantly on the urban course. The electrocardiogram analysis showed no noticeable change in the HF component time series for the urban course, although it tended to be higher on the Kodo course than the urban course. The LF/HF ratio was slightly higher for the urban course than for the Kodo course throughout.
Conclusions: The physiological and psychological measures indicate that walking for 2 hours in the forest is effective for relaxation.

Keywords: Kumano-Kodo forest; Walking; Relaxation; Cortisol; Amylase; POMS; Heart rate variability

\section{Introduction}

Human beings have existed for the past 600 to 700 million years. Over this time, more than $99.99 \%$ of humans have lived in natural environments, and their physiological functions are adapted to nature. Thus, people who live in contemporary urban and artificial environments tend to be constantly under stress. It has been suggested that forest-derived stimulation may reduce the stress of modern life [1]. Forest medicine research has been conducted in Japan since the 1990s. The Japanese term Shinrin-yoku, also called forest bathing or spending time in a forest environment, refers to the practice of incorporating the atmosphere of the forest into all of the senses. The first study to report that shinrin-yoku reduces stress was conducted in 1996 [2]. Many studies have since shown the stress-relieving effect of shinrin-yoku [3-7]. These studies measured cortisol in saliva, sympathetic and parasympathetic functions, blood pressure, and heart rate variability as indices of stress. Some studies have included walking in addition to spending time in forests, but with walking times as short as 15 minutes [8-12].

Kumano-Kodo, located in the south of the Kii Peninsula in Japan, has been a place of sacred pilgrimage since ancient times and its roads are used for daily life. There are many paths through the forest, some of which are paved due to the high rainfall. Kumano-Kodo was registered as a World Heritage site, "Sacred Sites and Pilgrimage Routes in the Kii Mountain Range," in 2007. The paths vary in elevation, and can be used 
as a hiking course according to each walker's physical strength The Japanese Ministry of Health, Labor and Welfare and Wakayama Prefecture recommend walking in Kumano-Kodo to reduce stress and improve physical fitness. This study examined the relaxation effect of walking on the Magose-Toge course, which is suitable for beginners as it has a relatively low height difference. Toge is the Japanese word for mountain pass. The walk is $4 \mathrm{~km}$ from the foot to the pass with a height difference of about $300 \mathrm{~m}$, and it takes about 2 hours to complete.

The purpose of this research is to investigate whether walking in forests for about 2 hours, using Kumano-Kodo Magose-Toge course, has a relaxing effect.

\section{Methods}

The experiment used an open crossover design. Twenty-two healthy male volunteers (average age $31.5 \pm 5.6$ years) were randomly assigned to two equal-sized groups. The aim and method of the study were explained, and informed consent was obtained from all participants. The study protocol was approved by the Ethics Review Committee for Human Experimentation of Mie University Graduate School of Medicine.

The participants completed two walks on consecutive weeks. The first group walked the Kodo (Kumano Kodo Magose-Toge) course first and the urban course second, while the second group walked them in the reverse order. The participants walked together as a group, and were not allowed to eat anything (including candy and gum) or drink more than the $500 \mathrm{ml}$ of drinking water provided per person.

On the Kodo course, the participants walked completed the $4.0 \mathrm{~km}$ walk from the foot to the Magose-Toge and bach in 2 hours, which took around two hours with breaks when necessary. On the first half of the urban course, which is flat and not surrounded by trees, the participants walked or jogged to increase their heart rate to 120 to 140 beats/min. This was assumed to be equivalent to climbing the Kodo course for 1 hour and 20 minutes. They then walked or jogged the second half of the course with a heart rate of 120 beats/ min, equivalent to the descent of the Kodo course. They walked and jogged for about 2 hours and covered about 7.8 $\mathrm{km}$ in total, with appropriate breaks as on the Kodo course. A pre-test was conducted to assess the average heart rate while walking the Kodo course; this was then used as the target heart rate for the urban course to ensure the exercise intensity was equal. The pre-test involved different participants from those in the main study, but they were of a similar age.

During the test, the weather, temperature, and humidity were measured and recorded at each location at the time of the participants' departure, midpoint, and arrival.

A watch-type heart-rate monitor (Accurex Plus, manufactured by Polar; disposable electrode vitrode $\mathrm{M}-150$ (G236), manufactured by Nihon Kohden) was attached to five patients in each group from the start of the walk to the end. The exercise load of both walking courses was analyzed.
Salivary amylase and salivary cortisol concentrations were measured. Salivary amylase and cortisol concentrations show diurnal variation, with a relatively steady period between 10 am and $4 \mathrm{pm}$ for the former and between 10:30 am and 5 pm for the latter. The tests were therefore conducted between 12:00 am and 4:00 pm. To further eliminate the influence of meals, participants were asked not to eat for one hour before the test. The saliva amylase concentration was measured after rinsing the mouth and then inserting a salivary amylase monitor chip (Nipro) under the tongue, then inserting the chip into the COCORO METER (Nipro) after 30 seconds. The measurements were made immediately before and immediately after walking before and after the walk started. A saliva sampling tube (Salivette, manufactured by Sarstedt) was used to collect saliva for the cortisol measurement. The attached cotton was placed under the tongue, and enough saliva was absorbed and returned to the sampling tube. The sampling tube was centrifuged at $3000 \mathrm{rpm}$ for 10 minutes and frozen and stored until the measurement.

For the psychological assessment, the participants completed the Japanese version of the Profile of Mood StatesBrief Form (POMS) [13] before and after walking. Scores for the "Tension-Anxiety", "Depression-Dejection", "AngerHostility", "Vigor", "Fatigue", and "Confusion" subscales were analyzed to indicate mood and emotion.

Participants indicated their general subjective physical and psychological condition at the start, midpoint (the Magose pass on the Kodo course, and the equivalent time on the urban course), and end of the walk by marking the appropriate place on a visual analog scale (VAS), with face indicators. The mark was then translated into a score for analysis.

To provide reference data, a Holter electrocardiograph (FM180, Fukuda Denshi) was attached to two participants from the first group and three from the second group from the start of the walk to the end. The low frequency (LF) and high frequency (HF) components were analyzed to assess autonomic nervous system activity. The measurement interval was 1 minute, the measurement algorithm was the maximum entropy method, and heart rate variability was assessed by power spectrum analysis.

For all data excluding electrocardiogram data, a variance analysis for crossover was carried out and t-test was carried out for all inter-group comparisons if there was no carry-over effect or order effect and it was significant. The POMS data, although parametric, were treated as nonparametric data, as is common in the field of psychology. No statistical analysis was carried out on the electrocardiogram waveform, because it was considered appropriate to look at the time series. For verification, SPSS Advanced Model version 13.0 was used, with a significance level of $5 \%$ for two-tailed tests.

\section{Results}

The analysis of variance for crossover indicated no carryover effect or order effect. It was concluded that the influence of the previous walking did not remain and the evaluation was not affected by the one week interval. 
The weather was fine on both walking days. Compared with the urban course, the Kodo course had a lower temperature and higher humidity, with humidity exceeding $60 \%$, which was somewhat uncomfortable. However, the difference was not considered sufficient to affect the comparison of the two courses.

The mean heart rate did not differ significantly between climbing up to the pass on the Kodo course $(118.90 \pm 4.59)$ and up to the midpoint of the urban course (117.3 \pm 3.93$)$. However, the mean heart rate was significantly higher $(p=0.018)$ on the descent of the Kodo course $(99.70 \pm 4.74)$ than on the second half of the urban course (108.90 \pm 3.84$)$. Overall, however, there was no significant difference (111.40 \pm 4.60 in the Kodo course, $114.00 \pm 3.73$ in the urban course; $p=0.337$ ) in heart rates, thus it was judged that the difference in momentum would not affect the comparison of the two courses.

The changes in salivary amylase concentrations are shown in Figure 1. On the Kodo course, the concentrations tended to be lower at the end of the walk than before the start, but the difference was not significant $(p=0.24)$. On the urban course, the mean concentration at the end of the walk was significantly $(p=0.027)$ higher than before the start, and significantly $(p=0.004)$ higher than at the end of the Kodo course. Although the relaxation effect of the Kodo course could not be confirmed, stress levels increased while walking the urban course, suggesting that the Kodo course was less stressful.

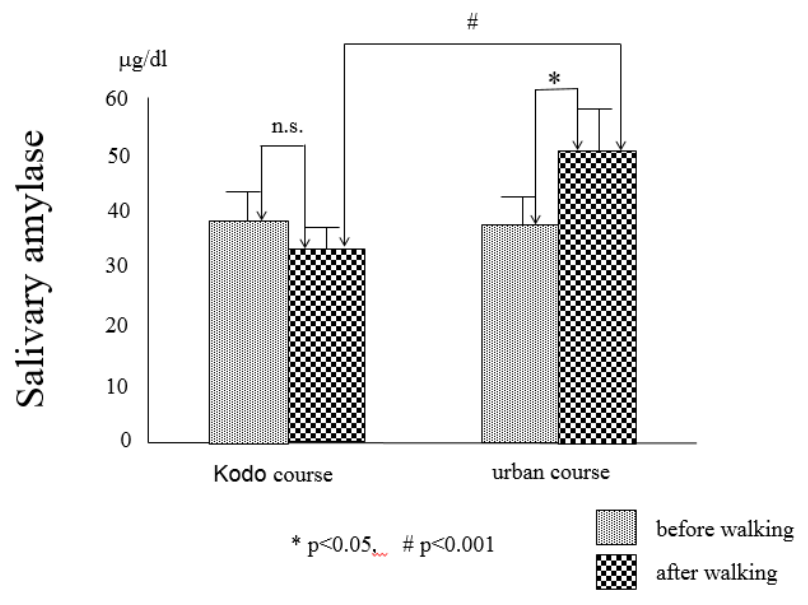

The data show mean \pm standard error. For all data, $n=22$

Figure 1 Salivary amylase concentrations before and after walking on the Kodo course and the urban course.

The changes in salivary cortisol concentration are shown in Figure 2. Salivary cortisol were lower at the end of both the Kodo course and the urban course than at the start. The difference was significant $(p=0.0001)$ for the Kodo course, although was not significance $(p=0.51)$ for the urban course. In the between-group comparison, cortisol levels tended ( $p$ $=0.081$ ) to be lower at the end of the Kodo course than at the end of the urban course. There was a tendency for participants to relax while walking, but the effect was higher for the Kodo course.

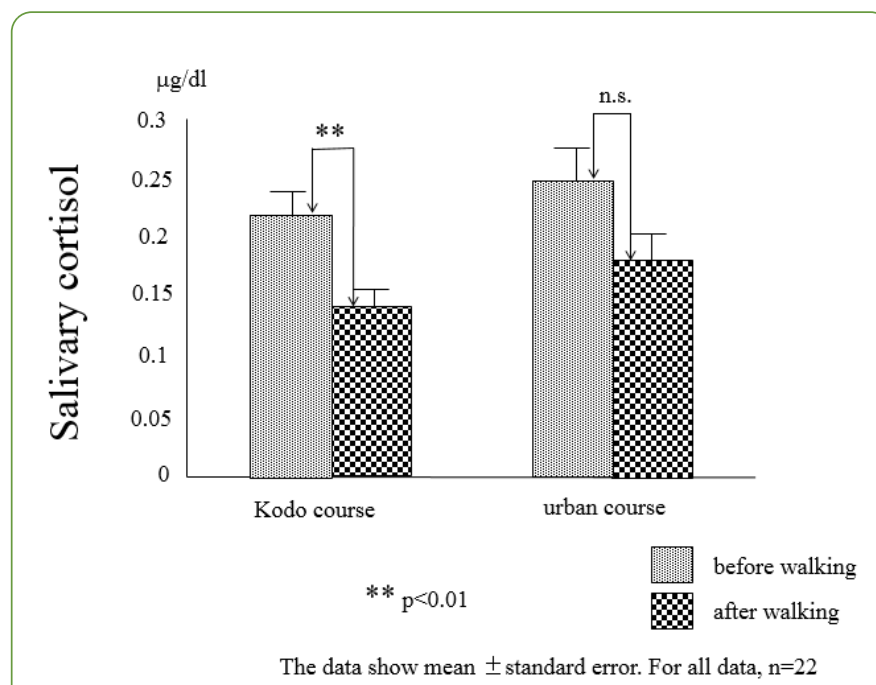

Figure 2 Salivary cortisol concentrations before and after walking on the Kodo course and the urban course.

The results of the POMS analysis are shown in Figure 3. "Tension-Anxiety" ( $p=0.0001$ in Kodo course; $p=0.02$ in urban course), "Depression-Dejection" ( $p=0.032$ in Kodo course; 0.042 in urban course), "Anger-Hostility" ( $p=003$ in Kodo course; $p=0.016$ in urban course), and "Confusion" ( $p=0.022$ in Kodo course; $p=0.042$ in urban course) significantly decreased after walking both the Kodo course and the urban course. Vigor" was significantly $(p=0.003)$ reduced on the urban course, and showed a declining trend $(p=0.057)$ compared to the Kodo course. "Fatigue" did not change on the Kodo course, but showed a tendency $(p=0.072)$ to increase on the urban course compared with the Kodo course. These results suggest that participants' mood improved while walking the Kodo course.

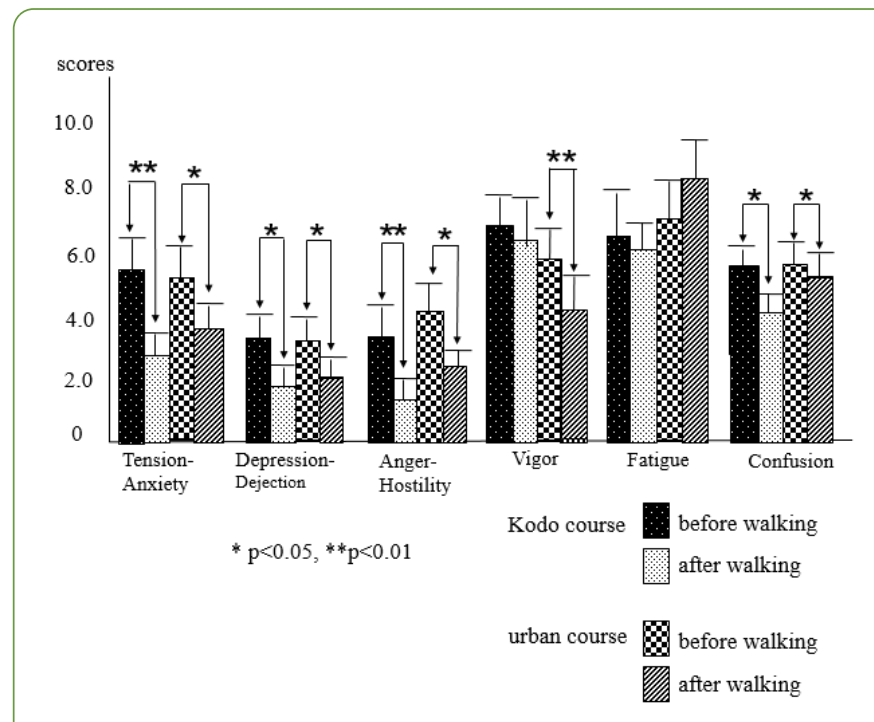

Figure 3 POMS scores before and after walking on the Kodo course and the urban course. 
For VAS analysis results, on the Kodo course, there was no change $(p=0.405)$ in scores at the midpoint, but scores were significantly $(p=0.005)$ higher at the end of the walk, indicating that participants were in a good mood and physical condition. On the urban course, however, scores were significantly $(p=0.008)$ lower at the midpoint and end of the walk, indicating that participants' mood and physical condition had worsened. A between-group comparison showed that scores on the urban course were significantly $(p=0.004)$ lower than on the Kodo course, both at the midpoint and end of the walk. Overall, these results suggest that the Kodo course improved the participants' overall mood.
Although the electrocardiogram analysis only provided reference data, the HF component, which is an indicator of parasympathetic activity, changed little from the initial value in the time series. On the Kodo course, however, the values were higher than on the urban course, especially near the end of the walk (Figure 4). The LF/HF ratio, which indicates the level of sympathetic nervous system activity, was somewhat higher on the urban course than the Kodo course throughout (Figure 5). These results suggest that the sympathetic nervous function was stable during the Kodo walk, but tended to be more activated during the urban walk.
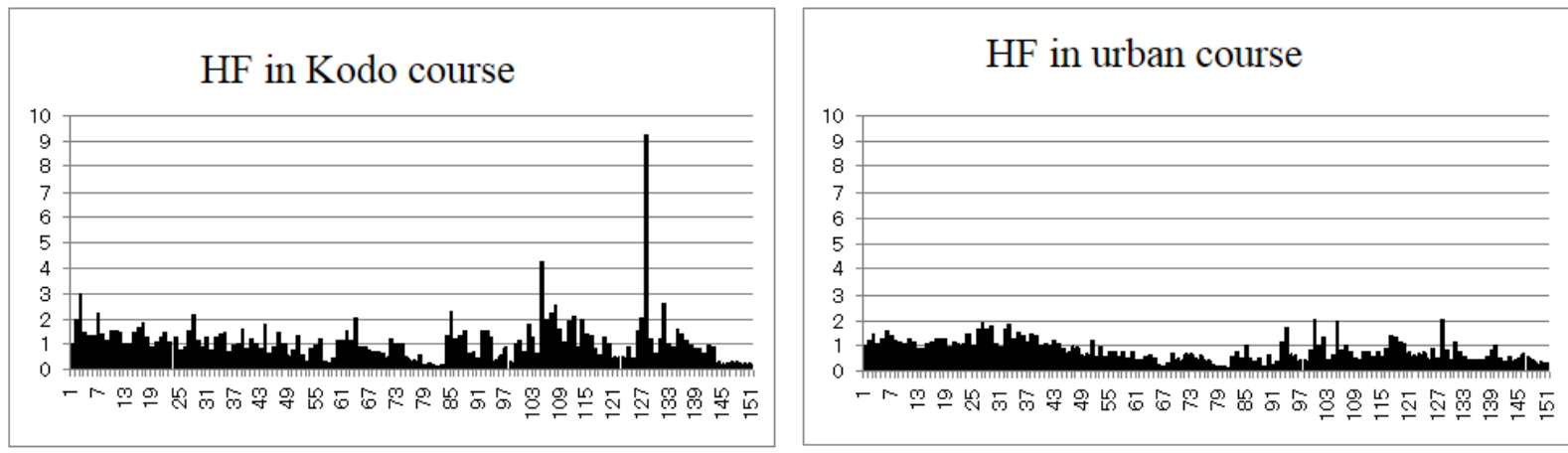

Average value of 5 subjects' data; Two out of 5 subjects walked on the Kodo course first, and the urban course second. The remaining 3 subjects walked them in the reverse order.

Figure $4 \mathrm{HF}$ component during walking in the Kodo course and in the urban course.
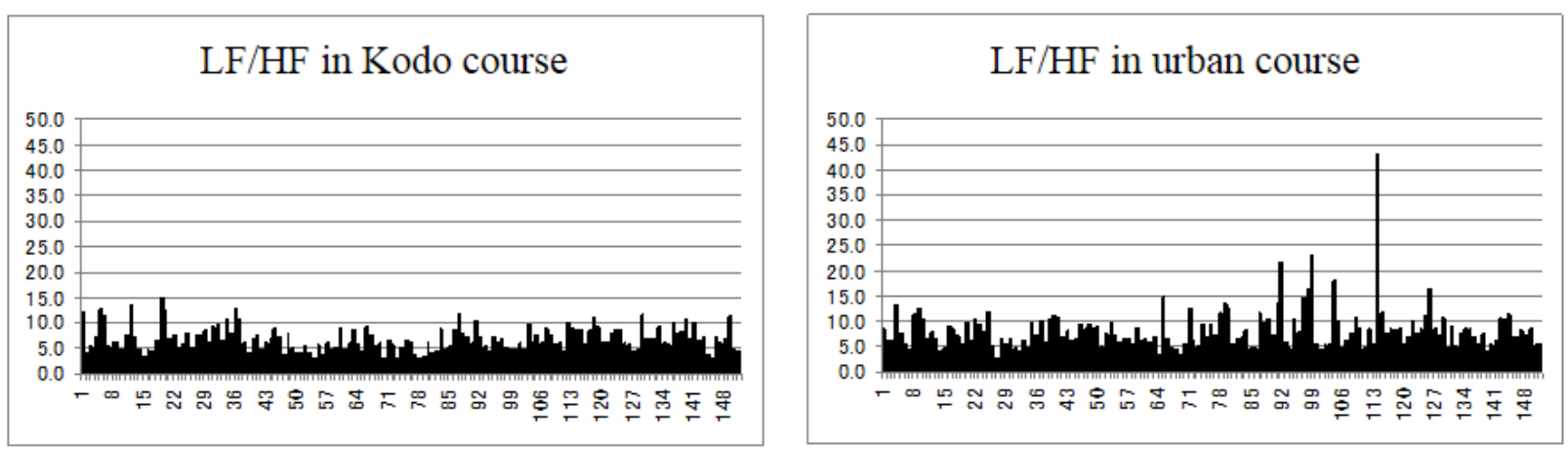

Average value of 5 subjects' data; Two out of 5 subjects walked on the Kodo course first, and the urban course second. The remaining 3 subjects walked them in the reverse order.

Figure 5 Changes of LF/HF ratio LF/HF ratio during walking in the Kodo course and in the urban course.

\section{Discussion}

Studies on forest bathing shinrin-yoku, most of which have been conducted in Japan [2-7], have shown that spending time in a forest reduces the pulse rate, reduces cortisol, suppresses sympathetic activity, increases parasympathetic activity, and lowers blood pressure compared with spending time in an urban area. Similar results are obtained even after only about 15 to 20 minutes of walking in the forest [8-12]. This highlights the importance of green spaces in the city [14] and garden walking [15]. However, the specific effects of the forest are not necessarily clear. A forest has a quiet atmosphere, beautiful scenery, mild climate, and fresh, clean air. Living in a forest environment for a long time may have a psychological effect or a physiological effect. Under the stress of restraint, strokeprone, spontaneously hypertensive rats exposed to phytoncides showed lower blood pressure than those without such exposure [16], indicating that phytoncides may have anti- 
stress effects. Phytoncides increase natural killer cell activity $[17,18]$. This favorable effect on immune functioning may in turn have a favorable psychoneuroimmunological influence. There are many cypress in Japan's forests, and the effect of the scent of hinoki, which is found on the Kodo course, can be considered part of the effect of forest bathing [19]. The visual sense of the forest has also been shown to have a relaxing effect [20]. The current study involved walking for a long time and distance, and found the relaxation effect conventionally shown for walking in a forest compared with urban areas. The characteristics of the forest must play a great part in explaining the effect.

It is well known that strong exercise produces sympathetic dominance, while moderate exercise results in parasympathetic dominance [21,22] and relieves stress if the subject is in training [23]. However, it is unclear how much exercise is moderate. Although the participants of this study had no particularly training, it is likely that the intensity of the exercise caused the relaxation effect, enhanced by the forest environment.

Stress response is mainly caused by sympathetic nervous system and hypothalamus-pituitary-adrenal (HPA) axis. Salivary amylase and salivary cortisol concentrations reflect sympathetic nervous function and HPA function, respectively, and they are used as a useful index because they are noninvasive [24]. To measure the sympathetic nervous function, heart rate variability that was used in this experiment has come to be used frequently. Relaxation is thought to be a state in which both sympathetic and HPA functions are suppressed. On the Kodo course both functions showed a suppression or suppression tendency, while in the urban course the HPA function showed a tendency to suppress, but the sympathetic nervous function was not suppressed. Therefore we have concluded that the relaxation effect was recognized in the Kodo course, but not adequately in the urban course.

The Kodo path includes some stone pavement, and is not flat like an urban course. It is necessary to retain the body's balance during walking, which uses muscles that are not used in urban walking. It is also thought that there are many nerve connections between the foot and the brain that are highly stimulated by walking [25]. Whether these factors lead to relaxation has not yet been examined, but it is possible that they make some contribution, and further study is required.

Kumano Kodo is a prayer path, which may have had an effect, although this is impossible to prove and it is better interpreted as an effect of the forest.

The results of this study suggest that walking for 2 hours has a relaxing effect that depends on the exercise intensity. As walking can also strengthen physical fitness, the use of the forest for improving health seems to be an excellent suggestion. The relationship between humans and nature in terms of stress relief, health promotion, rehabilitation, and disease prevention would be worthwhile [26]. We aim to conduct further research on how walking in the forest contributes to health. It is also necessary to investigate reproducibility by increasing the number of subjects, examination targeting women, and studies that broaden target age in the future.

\section{Conclusion}

This study provides psychological and physiological evidence that confirms the relaxing effect of walking in a forest. Although previous studies have reported this effect, their results were based on walking for only about 15 minutes. The present study shows, for the first time, the relaxation effect of walking for as long as 2 hours in a forest. Walking for this length of time is beneficial not only for relaxation, but also for improving physical fitness. Further research is required to clarify the contribution of factors such as the intensity of exercise. It is also necessary to increase the number of subjects and to examine the effects of sex and age.

\section{Acknowledgements}

We are very grateful to all of the volunteers who made this study possible.

\section{Funding}

This research was conducted in collaboration with the Mie Technology Licensing Organization and received financial support from Owase City and its related organizations.

\section{Disclosure of Interests}

The authors report no conflicts of interest.

\section{References}

1. Song C, Ikei H, Miyazaki Y (2016) Physiological effects of nature therapy: a review of the research in Japan. Int J Environ Res Public Health 13 pii: E781.

2. Miyazaki Y, Motohashi $Y$ (1996) Forest environment and physiological response. In: Agishi $\mathrm{Y}$, Ohtsuka $\mathrm{Y}$, (eds). New Frontiers in Health Resort Medicine. Sapporo: Hokkaido School of Medicine Press Pp: 67-77.

3. Lee J, Park BJ, Tsunetsugu Y, Ohira T, Kagawa T, et al. (2011) Effect of forest bathing on physiological and psychological responses in young Japanese male subjects. Public Health 125: 93-100.

4. Park BJ, Tsunetsugu Y, Lee J, Kagawa T, Miyazaki Y (2012) Effect of the forest environment on physiological relaxation-the results of field tests at 35 sites throughout Japan. In: Li Q, editor. Forest Medicine. New York: Nova Science Publishers pp: 55-65.

5. Calogiuri G, Chroni S (2014) The impact of the natural environment on the promotion of active living: an integrative sysytemic review. BMC Public Health 14: 873.

6. Deymour V (20169 The human-nature relationship and its impact on health: a critical review. Front Public Health.

7. Park BJ, Tsunetsugu Y, Kasetani T, Kagawa T, Miyazaki Y (2010) The physiological effects of Shinrin-yoku (taking in the forest 
atmosphere or forest bathing): evidence from field experiments in 24 forests across Japan. Environ Health Prev med 15: 18-26.

8. Tsunetsugu $Y$, Park BJ, Ishii $H$, Hirano $H$, Kagawa T, et al. (2007) Physiological effects of "Shinrin-yoku" (taking in the atmosphere of the forest) in an old-growth broadleaf forest in Yamagata prefecture, Japan. J Physiol Anthropol 26: 135-142.

9. Tsunetsugu $\mathrm{Y}$, Park BJ, Miyazaki $\mathrm{Y}$ (2010) Trends in research related to "Shinrin-yoku" (taking in the forest atmosphere or forest bathing) in Japan. Environ Health Prev Med 15: 27-37.

10. Lee J, Tsunetsugu Y, Takayama N, Park BJ, Li Q, et al. (2014) Influence of forest therapy on cardiovascular relaxation in young adults. Evid. Based Complement. Altern Med Article ID 834360.

11. Song C, Ikei H, Igarashi M, Takagi M, Miyazaki Y (2015) Physiologocal and psychological effects of a walk in urban parks in fall. Int J Environ Res Public Health 12: 14216-14228.

12. Song C, Ikei H, Kobayashi M, Miura T, Taue M, et al. (2015) Effect of forest walking on autonomic nervous system activity in middle-aged hypertensive individuals: a pilot study. Int J Environ Res Public Health 12: 2687-2699.

13. Jacobsen PL, Mahableshwarkar AR, Chen Y, Chrones L, Clayton $\mathrm{AH}$ (2015) Effect of vortioxetine vs. escitalopram on sexual functioning in adults with well-treated major depressive disorder experiencing SSRI-induced sexual dysfunction. J Sex Med 12: 2036-2048.

14. Irvine KN, Warber SL, Devine WP, Gaston KV (2013) Understanding urban green space as a health resource: a qualitative comparison of visit motivation and derived effects among park users in Sheffield, UK. Int J Environ Res Public Health 10: 417-442.

15. McCaffrey R, Liehr P (2016) The effect of reflective garden walking on adults with increased levels of psychological stress. J Holist Nurs 34: 177-84.

16. Kawakami K, Kawamoto M, Nomura M, Otani H, Nabika T, et al. (2004) Effects of phytoncides on blood pressure under restraint stress in SHRSP. Clin Exp Phamacol Phsiol 31: S27-S28.
17. Li Q, Kobayashi $M$, Wakayama $Y$, Inagaki $H$, Katsumata $M$, et al. (2009) Effect of phytoncide from trees on human natural killer cell function. Int J Immunopathol Pharmacol 22: 951-959.

18. Li Q, Kawada T (2011) Effect of forest environments on human natural killer (NK) activity. Int J Immunopathol Pharmacol 24: 39S-44S.

19. Ikei H, Song C, Miyazaki Y (2015) Physiological effect of olfactory stimulation by Hinoki cypress (Chamaecyparis obtusa) leaf oil. J Physiol Anthropol 34: 44.

20. Igarashi M, Yamamoto T, Lee J, Song C, Ikei H, et al. (2014) Effects of stimulation by three-dimensional natural images on prefrontal cortex and autonomic nerve activity: a coparison with stimulation using two-diensional images. Cogn Process 15: 551-556.

21. Yamamoto Y, Hughson RL, Peterson JC (1991) Autonomic control of heart rate during exercise studied by heart rate variability spectral analysis. J Appl Physiol 71: 1136-1142.

22. Iwane $M$, Arita $M$, Tomimoto $S$, Satani $O$, Matsumoto $M$, et al. (2000) Walking 10,000steps/day or more reduces blood pressure and sympathetic nerve activity in mild essential hypertension. Hypertens Res 23: 573-580.

23. Zschucke E, Renneberg B, Dimeo F, Wüstenberg T, Ströhle A (2015) The stress-buffering effect of acute exercise: evidence for HPA axis negative feedback. Psychoneuroendocrinology 51: 414-425.

24. Schmacher S, Kirschbaum C, Fydrich T, Ströhle A (2013) Is salivary alpha-amylase an indicator of autonomic nervous system dysregulations in mental disorders?-A review of preliminary findings and the interactions with cortisol. Psychoneuroendocrinology 38: 729-743.

25. Embong NH, Soh YC, Ming LC, Wong TW (2015) Revisiting reflexology: concept, evidence, current practice, and practiotioner. J Tradit Complement Med 5: 197-206.

26. Seymour $V(2016)$ The human-nature relationship and its impact on health: a critical review. Front Public Health 4: 259-260. 